A review of recent developments on turbulent entrainment in stratified flows

This article has been downloaded from IOPscience. Please scroll down to see the full text article.

2010 Phys. Scr. 2010014044

(http://iopscience.iop.org/1402-4896/2010/T142/014044)

View the table of contents for this issue, or go to the journal homepage for more

Download details:

IP Address: 141.211.173.82

The article was downloaded on 08/08/2011 at 19:45

Please note that terms and conditions apply. 


\title{
A review of recent developments on turbulent entrainment in stratified flows
}

\author{
Aline J Cotel \\ Department of Civil and Environmental Engineering, University of Michigan, Ann Arbor, \\ MI 48109-2125, USA \\ E-mail: acotel@umich.edu
}

Received 26 October 2009

Accepted for publication 24 March 2010

Published 31 December 2010

Online at stacks.iop.org/PhysScr/T142/014044

\begin{abstract}
Stratified interfaces are present in many geophysical flow situations, and transport across such an interface is an essential factor for correctly evaluating the physical processes taking place at many spatial and temporal scales in such flows. In order to accurately evaluate vertical and lateral transport occurring when a turbulent flow impinges on a stratified interface, the turbulent entrainment and vorticity generation mechanisms near the interface must be understood and quantified. Laboratory experiments were performed for three flow configurations: a vertical thermal, a sloping gravity current and a vertical turbulent jet with various tilt angles and precession speeds. All three flows impinged on an interface separating a two-layer stably stratified environment. The entrainment rate is quantified for each flow using laser-induced fluorescence and compared to predictions of Cotel and Breidenthal (1997 Appl. Sci. Res. 57 349-66). The possible applications of transport across stratified interfaces include the contribution of hydrothermal plumes to the global ocean energy budget, turbidity currents on the ocean floor, the design of lake de-stratification systems, modeling gas leaks from storage reservoirs, weather forecasting and global climate change.
\end{abstract}

PACS numbers: 47.55.Hd, 47.55.P-

(Some figures in this article are in colour only in the electronic version.)

\section{Introduction}

Transport across a stratified interface is an essential aspect of many geophysical processes. Applications of it include pollution transport in urban areas, where strong inversions are a common phenomenon (e.g. Salt Lake City) and therefore katabatic winds are essential in dispersing highly concentrated pollutants; or the design of sewage outfalls in urban areas, such as the newly redesigned Boston sewage outfall that takes advantage of summer stratification to trap effluents below the water surface. To start solving these issues, one must begin with a profound understanding of turbulent mixing, entrainment and vortex dynamics at the interface. Entrainment is the first step in turbulent mixing, and turbulent mixing, in turn, is an essential factor in the processes described above. Once one is able to quantify the physical processes at the interface, more accurate transport models can be developed.

Cotel and Breidenthal (1997) proposed a theoretical model for predicting the turbulent entrainment rate across a stratified interface over a wide range of Reynolds, Richardson and Schmidt numbers. A new parameter was proposed: persistence, defined as the ratio of rotational to translational velocity associated with entraining eddies in the vicinity of the interface. To extend and further test the validity of the persistence parameter, laboratory experiments were performed for a tilted jet with and without precession and compared to experiments with a vertical jet impinging on a stratified interface (Cotel 1995). Furthermore, the effect of a solid boundary is examined by investigating a sloping gravity current impinging on a stratified interface. The importance of the forcing mechanism, i.e. momentum versus buoyancy, is investigated by studying thermals in a stratified environment.

\section{A turbulent jet impinging on a stratified interface}

\subsection{Experimental setup and procedure}

A water tank, $28 \times 28 \times 60 \mathrm{~cm}$ high, was divided by a horizontal sliding splitter plate into two regions. The reservoir was stably stratified with the lower layer composed of a 
saline solution mixed with fluorescein dye and the upper layer composed of a lower-density saline solution, whose density varied depending on the experiments. Once the fluids settled, i.e. once the turbulence due to the filling process had dissipated, the splitter plate was slowly withdrawn, leaving a thin, stratified interface between the two fluids of thickness equal to $0.3 \mathrm{~cm}$. The valve located in the vicinity of the jet nozzle was opened to start the experiment. Different nozzles have been constructed to provide a range of different tilt angles. The nozzle was attached to a disc that can be rotated at different speeds using a standard dc motor.

\subsection{Results}

Laser-induced fluorescence (LIF) and particle image velocimetry (PIV) measurements were carried out for a tilted jet at two different angles, $5^{\circ}$ and $10^{\circ}$, and compared with previous data for $15^{\circ}$ (Cotel et al 1997). For a tilt angle of $5^{\circ}$, the range of the Richardson number was from 0.2 to 20.8 and the Reynolds number was in the range between 7520 and 11700 . For a tilt angle of $10^{\circ}$, the range of the Richardson number was narrowed to the region of interest, between 1.2 and 8.6. A previous work (Cotel 1995) has shown a transition around $R i=10$, where the entrainment process changes from an engulfment-driven mechanism to a diffusion-dominated process. The entrainment measurements were carried out soon after the impingement. The internal waves produced at the interface during impingement did not interfere with the dynamics of the flow since no breaking of the internal waves was observed. Furthermore, the interface between the jet and surrounding fluid was sharp; diffusion does not play a significant role in the entrainment process for the range of $R i$ investigated.

The entrainment rate (the ratio of entrainment velocity to jet velocity at the interface; Cotel and Breidenthal 1997) was measured for a jet at different tilt angles. The Richardson number was defined using the jet quantities and the initial density difference across the interface; it is defined as

$$
R i=\frac{g^{\prime} \delta}{w_{1}^{2}}
$$

where $g^{\prime}$ is the buoyancy acceleration due to the density difference $\Delta \rho$ :

$$
g^{\prime}=\frac{\Delta \rho}{\rho} g .
$$

The acceleration of gravity is $g . \Delta \rho$ is the density difference across the interface. The density of the surrounding fluid is $\rho . \delta$ is the length scale of the incident turbulence at the interface and $w_{1}$ is its characteristic velocity. Figure 1 shows the measured entrainment rates (and error bars) as a function of $R i$ for different jet tilt angles. For angles up to $10^{\circ}$, the entrainment rate follows the same power law, $R i^{-1 / 2}$ whereas for the $15^{\circ}$ case, the entrainment rate is proportional to $R i^{-3 / 2}$. There is no significant difference in the data for the $5^{\circ}$ and $10^{\circ}$ cases. There appears to be a significant change in the jet dynamics at an angle between $10^{\circ}$ and $15^{\circ}$, which happens to be the half-angle of expansion for a vertical turbulent jet (Dahm and Dimotakis 1987).

A vertical jet impinging on a stratified interface exhibits the following structure: on each side of the impingement

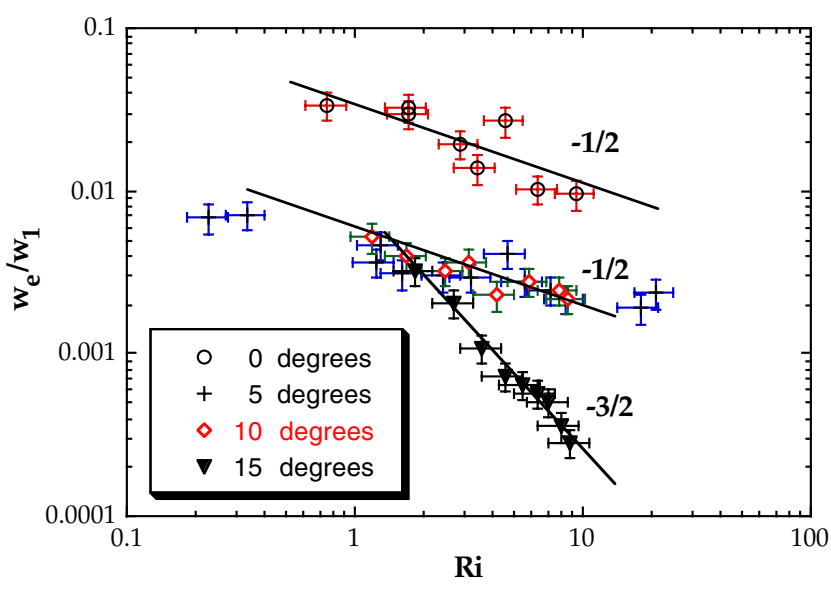

Figure 1. Entrainment rate versus Richardson number for different tilt angles.

(a)

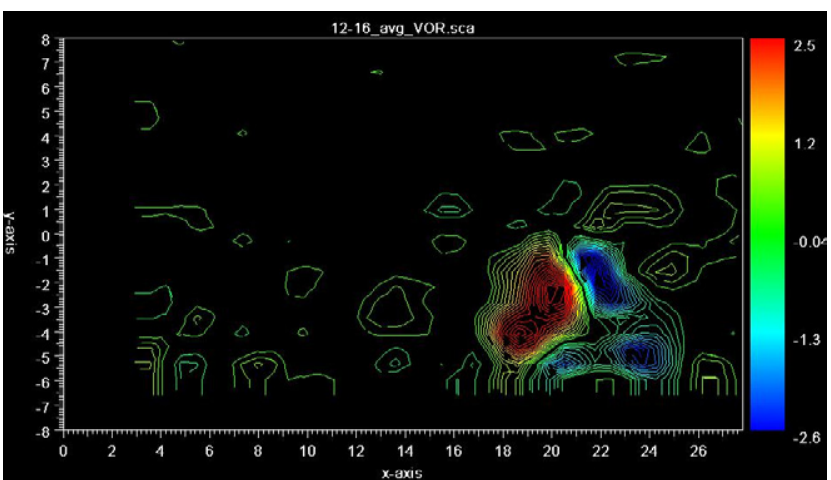

(b)

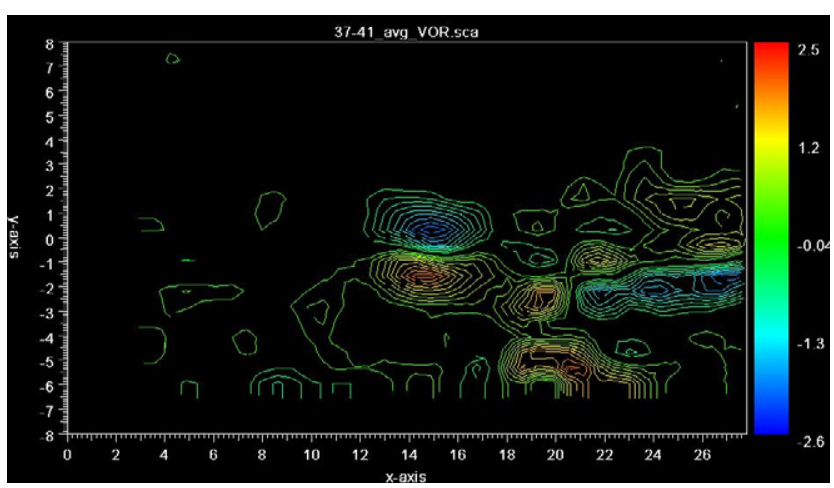

Figure 2. Vorticity contours $\left(\mathrm{s}^{-1}\right)$ from PIV data for a $10^{\circ}$-tilted jet at $R i=8.7$. The $x$ and $y$ axes represent the horizontal and vertical axes of the apparatus (cm). (a) Before impingement. (b) After impingement, note the formation of baroclinic vortices, rotating in the opposite direction to the jet vortices only on one side.

dome, a strong baroclinic vortex is formed, and that vortex is responsible for entraining and mixing the surrounding fluid (Cotel 1995). For a tilt angle of $15^{\circ}$, this vortical structure is not fully formed; only one baroclinic vortex completely rolls up and is strong enough to entrain fluid, while the opposite side shows much weaker baroclinic vorticity. Figure 2(a) shows the vorticity contours for $R i=8.7$ and for a tilt angle of $10^{\circ}$ before impingement. The jet vortices are clearly shown in red and blue, representing the two signs of vorticity within the jet structure. In figure 2(b), which represents the after-impingement situation, there is a baroclinic vortex on 


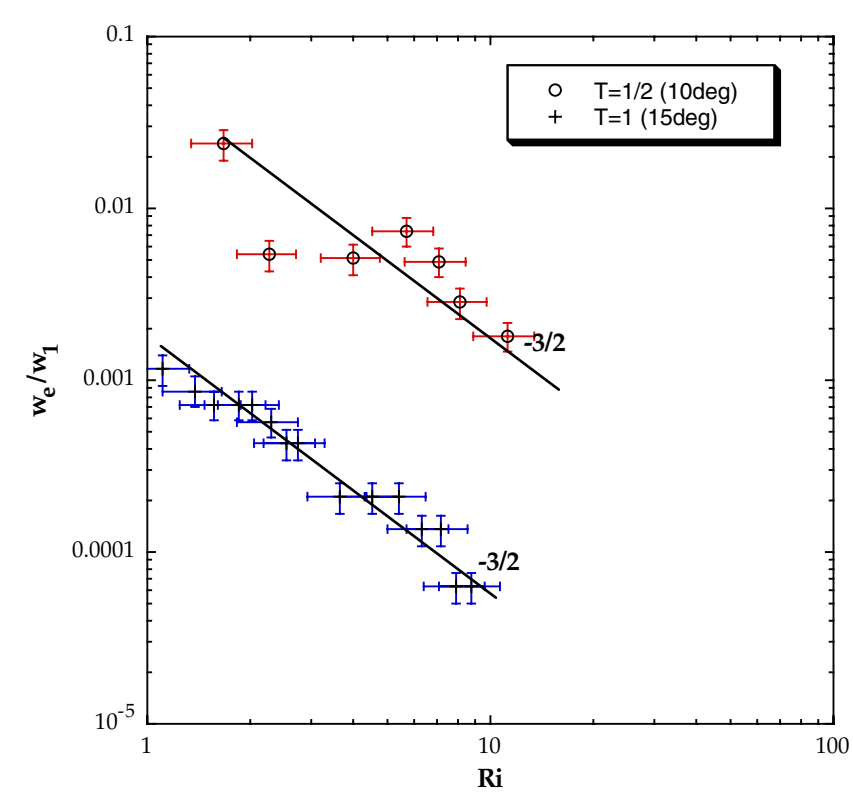

Figure 3. Effect of the persistence parameter on the entrainment rate for a tilt angle of $10^{\circ}$, compared to a tilt angle of $15^{\circ}$.

the left side located just above the jet vortex, rotating in the opposite direction to the jet vortex. However, on the right side of the image, there is no distinct baroclinic vortex above the jet vortex. Even at that angle, the baroclinic vorticity starts changing its dynamics and is not able to roll up completely and form vortices around the impingement dome. Figure 2(b) shows an average of five image pairs, representing an average over $0.1 \mathrm{~s}$ given our PIV data acquisition conditions.

\subsection{Effect of precession}

A dc motor was installed in order to provide a means to rotate the jet nozzle at various angular velocities. The goal of this set of experiments was to determine the transition between entrainment regimes by only changing the vortex persistence parameter, i.e. the precessing speed. Figure 3 compares the entrainment rate as a function of Richardson numbers for different values of the vortex persistence parameter. With no precession, a $10^{\circ}$-tilted jet follows an $R i^{-1 / 2}$ power law. For low values of the precessing speed, no clear pattern is observed, and a significant amount of scatter is present. The entrainment is in between persistent and non-persistent regimes. However, for a persistence parameter equal to $1 / 2$, we found that the entrainment rate is consistently in the non-persistent regime, proportional to $R i^{-3 / 2}$. It is possible to physically force a persistent regime into a non-persistent regime by imposing a large enough precessing speed. Precession is a valuable tool when a low entrainment rate is desirable in a particular situation; by manipulating the persistence parameter, a reduction of the order of two orders of magnitude in the entrainment rate can be achieved.

\subsection{Discussion}

There is a direct relationship between the entrainment rate for a tilted jet impinging on a stratified interface and the vortical structure surrounding the impingement dome. When the baroclinic vortices are stationary, the entrainment rate follows an entrainment law (proportional to $R i^{-1 / 2}$ ) characteristic of a persistent regime, as defined by Cotel and Breidenthal (1997). The transition between persistent and non-persistent regimes occurs at an angle between $10^{\circ}$ and $15^{\circ}$, which is equivalent to the half-angle of the jet. When the tilt angle is greater than the critical half-angle of the jet, the entrainment rate always exhibits a non-persistent behavior. In other words, the baroclinic vortices created at impingement are either weak or non-existent. However, when the tilt angle is smaller than the half-angle of expansion, most of the baroclinic vortices around the impingement dome complete a full rotation and contribute greatly to entrainment.

At lower tilt angles, it is possible to reduce the entrainment rate by imposing a large enough precessing speed. A naturally persistent regime is then forced into a non-persistent entrainment regime, thus providing a much lower entrainment for the same values of Richardson number.

\section{Sloping gravity currents impinging on a stratified interface}

Details of the experimental setup and procedure are provided in Samothrakis and Cotel (2006) and a detailed literature review is also provided there. Our present objective is to test the persistence theory of Cotel and Breidenthal (1997) against these recent results.

Two types of experiments were performed: constant flux and finite volume.

\subsection{Results}

3.1.1. Constant flux. The velocity of the gravity current, $U_{f}$, compares very well with previous studies (Britter and Linden 1980). This shows that the gravity current is well developed when it hits the interface and the effect of friction is negligible. Two mechanisms were found to be responsible for mixing. The first one is the overriding and engulfment of ambient fluid beneath the nose of the head. There is no mixing at the front of the current. Large eddies are responsible for entraining the surrounding fluid and bringing it to the center of the gravity current head, where it is ultimately mixed. The second mixing mechanism is due to the Kelvin-Helmholtz instabilities along the upper surface of the head. The rate of growth of the head $\mathrm{d} h / \mathrm{d} x$ was measured using planar laser-induced fluorescence, with $h$ being the height of the head and $x$ being the downstream distance along the slope. This ratio was measured at two different locations to ensure that the effect of the interface on the entrainment of the gravity current was considered. The rate of growth of the head at the two locations is found to be proportional to $R i^{-1 \pm 0.1}$ (Samothrakis and Cotel 2006).

3.1.2. Finite volume. The same two mechanisms responsible for mixing were also observed for the case of a finite-volume gravity current: overriding of ambient fluid by the gravity current head and Kelvin-Helmholtz instabilities on the upper surface of the head. The Kelvin-Helmholtz vortices represented the dominant entrainment and mixing mechanism responsible for the bulk of the mixing. Using planar laser-induced fluorescence, pixel values were calibrated as a 
function of their mixing ratio (Samothrakis 2005). The ratio of the volume of mixed fluid, $V_{\text {mix }}$, to the initial volume of the gravity current, $V_{\mathrm{o}}$, is found to be proportional to $R i^{-1 \pm 0.15}$. The rate of growth of the head of a finite-volume gravity current is found to be proportional to $R i^{-1 \pm 0.1}$. The $R i$ number governing the entrainment and mixing rates is based on the difference between the current and the bottom layer.

3.1.3. Discussion. For both the cases of a constant-flux gravity current and a finite-volume gravity current impinging on a stratified interface, the Kelvin-Helmholtz vortices, formed on the upper surface of the head of the gravity current, represented the controlling mechanism for entrainment and mixing, which are both proportional to $R i^{-1}$. This corresponds to a shear-dominated regime and was predicted by Cotel and Breidenthal (1997).

\section{Thermal/plume impinging on a stratified interface}

The term 'thermal' is used to denote a parcel of isolated buoyant fluid suddenly released from rest, which subsequently moves under the action of buoyancy forces alone. Details of the experimental setup and a detailed literature review are provided in Cotel and Kudo (2008). According to Cotel and Breidenthal (1997), a thermal should behave similarly to a vortex ring if one assumes that persistence controls entrainment. The fundamental difference between these two flows is in the forcing mechanism, momentum versus buoyancy. By comparing recent results from Cotel and Kudo (2008) to predictions from Cotel and Breidenthal (1997), one can ascertain the importance of the persistence versus forcing mechanism.

\subsection{Results}

Instead of behaving similarly to a vortex ring (Linden 1973), i.e. rebounding away from the interface, two vortices are created by baroclinic torque from the tilted interface; then these vortices merge with the original thermal vortices to form a vortex pair moving horizontally in the vicinity of the interface (Cotel and Kudo 2008). No significant vertical transport was observed. Zhang and Cotel (2000) measured the entrainment rate of a single thermal impinging on a stratified interface and found it to be proportional to $R i^{-3 / 2}$ as predicted by Cotel and Breidenthal's (1997) persistence theory. Therefore the forcing mechanism has absolutely no bearing on the entrainment process; only the value of the persistence parameter determines the entrainment rate for the same range of $R e, R i$ and $S c$.

\section{Conclusions}

The new results presented above confirm the wide applications of the persistence theory of Cotel and
Breidenthal (1997). In the case of the sloping gravity current, shear, through the action of Kelvin-Helmholtz vortices, dominates the entrainment process and therefore an $R i^{-1}$ power law is observed. For buoyancy-driven flows such as thermals, the persistence theory applies directly, no stationary eddies are created along the impingement dome and the baroclinic eddies control the entrainment, which is proportional to $R i^{-3 / 2}$. The forcing mechanism, i.e. buoyancy or momentum, has no effect on the entrainment rate; the vortical structure resulting from the impingement is responsible for the type of entrainment that takes place. The ultimate test for the persistence theory is provided by the precessing and tilted jet experiments. The vortical structure of the jet is deeply affected by the tilt angle of the jet. A transition between persistent and non-persistent regimes is observed for a tilt angle between $10^{\circ}$ and $15^{\circ}$. A naturally occurring persistent regime can be forced into a non-persistent regime by imposing a sufficiently large precessing speed, which corresponds to a critical value of the persistence parameter for the jet. As a result, the entrainment rate of the jet is reduced by at least one order of magnitude for the same values of the Richardson, Reynolds and Schmidt numbers.

The turbulent entrainment rate is predicted and quantified for a variety of flow conditions and a wide range of parameters, thus allowing for a direct quantification of turbulent mixing for the same conditions since as previously noted (Cotel and Breidenthal 1997), entrainment and turbulent mixing are proportional to one another for Reynolds numbers above the mixing transition (2000-3000).

\section{References}

Britter R E and Linden P F 1980 The motion of the front of a gravity current traveling down an incline J. Fluid Mech. 99 531-43

Cotel A J 1995 Entrainment and detrainment of a jet impinging on a stratified interface PhD Thesis University of Washington, Seattle, WA

Cotel A J and Breidenthal R E 1997 A model of stratified entrainment using vortex persistence Appl. Sci. Res. 57 349-66

Cotel A J, Gjestvang J A, Ramkhelawan N N and Breidenthal R E 1997 Laboratory experiments of a jet impinging on a stratified interface Exp. Fluids 23 155-60

Cotel A J and Kudo Y 2008 Impingement of buoyancy-driven flows on a stratified interface Exp. Fluids 45 131-9

Dahm W J A and Dimotakis P E 1987 Measurement of entrainment and mixing in turbulent jets AIAA J. 25 1216-23

Linden P F 1973 The interaction of a vortex ring with a sharp density interface J. Fluid Mech. 60 467-80

Samothrakis P 2005 An experimental investigation of the dynamics of a sloping gravity current impinging on a stratified interface PhD Thesis The University of Michigan, Ann Arbor, MI

Samothrakis P and Cotel A J 2006 The propagation of a gravity current in a two-layer stratified environment J. Geophys. Res. $111 \mathrm{C} 01012$

Zhang Q and Cotel A J 2000 Entrainment due to a thermal impinging on a stratified interface with and without buoyancy reversal J. Geophys. Res. 105 15457-67 